\title{
2000 国家大地坐标系转换方法及其质量控制
}

李兆黎

安庆市勘察测绘院

DOI:10.32629/gmsm.v2i6.402

[摘 要] 为推进地理信息数据的应用与共享,提高地理信息数据服务水平,需要将原有坐标系地理信息数据转换到2000国家大地坐标系。本文就 结合安庆市2000国家大地坐标系转换工作实例,对大地坐标系转换方法及其质量控制技术展开探讨,希望能为相关人士提供些许参考。

[关键词] 2000 国家大地坐标系; 转换; 质量控制

2008年国家测绘地理信息局发布第2号公告 “根据《中华人民共和国 测绘法》, 经国务院批准, 我国自2008年7月1日起, 启用2000国家大地坐标 系” (简称CGCS2000)。公告规定了2000国家大地坐标系与现行国家大地坐 标系转换、衔接的过渡期为 8 年至 10 年。现有各类测绘成果, 在过渡期内可 沿用现行国家大地坐标系; 2008年7月1日后新生产的各类测绘成果应采用 2000国家大地坐标系。同时现有地理信息系统, 在过渡期内应逐步转换到 2000国家大地坐标系；2008年7月1日后新建设的地理信息系统全应采用 2000 国家大地坐标系, 这就需要对原有坐标系进行转换, 从而更好地构建 地理信息数据的应用与共享机制。

\section{1 安庆市原有坐标系情况}

1.1安庆独立坐标系。2008年安庆市控制网改造扩建项目中, 平面控制 主网以总参 II 等补网点风凰山、井头山为起算点, 进行约束平差, 由于没有 更多的已知点做为检核, 故平面系统称为安庆市独立坐标系。

1.21954 年北京坐标系。中央子午线 $117^{\circ}, 3$ 度带 (带号39) 高斯投影, 横坐标加 $500 \mathrm{~km}$ 。

1.31980 西安坐标系。国土数据主要为1980西安坐标系。

\section{0 国家大地坐标系的转换方法}

2.1 观测点位设计。由于缺少 2000 国家大地坐标系控制点成果数据, 为了构建安庆市现有坐标系与 2000 国家大地坐标系的转换关系, 需均匀选 择至少 6 个安庆市控制点进行联测。

根据点位普查结果, 从2008年安庆市测绘控制网改造扩建项目的51个 点中至少选择可利用的13个点JTSH、R636、R685、R686、R925、R926、R927、 GD11、GD14、GD27、GD31、GD41、GD46, 通过GNSS观测, 解算得到其2000 国家大地坐标系坐标。

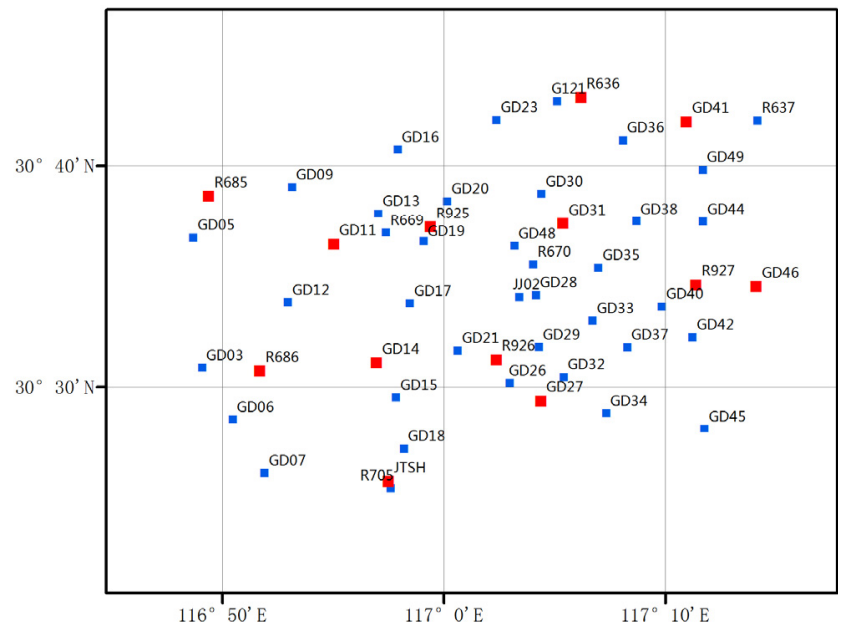

图1 安庆市基础控制网点布设图
2.2数据的观测。GNSS C级网观测采用双频大地型GNSS接收机, 标称精 度不低于 $5 \mathrm{~mm}+1 \mathrm{ppm}$ 。采用基于连续运行基准站点观测模式。观测前, 作业 所用的GNSS接收机及天线应送国家计量部门认可的仪器检定单位检定, 检 定合格且在有效期限内方能在观测作业中使用。

2. 3数据下载与存储。(1) 每一个观测时段的观测数据应及时下载, 下 载之后应立即将原始观测数据转换为RINEX格式数据, 以检查原始数据下 载是否正确, 下载、转换软件使用接收机随机软件; (2) 每天的原始观测数 据使用一个子目录, 子目录命名方式为: 测站编号十年代十该天的年积日 $+\mathrm{D}$ (表示原始观测数据)。(3) 每天的RINEX数据使用另一个目录, 该子目录 命名方式为: 测站编号 + 年代 + 该天的年积日 $+R$ (表示RINEX格式数据)。 (4) 原始数据与RINEX数据必须在微机硬盘中保留到上交的数据检查验收 完成后, 并在不同的介质上备份。

\section{3 坐标转换参数计算}

3. 1 重合点分析与确定。当两种不同坐标系进行转换时, 坐标转换的 精度除了取决于坐标转换的数学模型和求解转换参数的公共点 (重合点) 坐标精度外, 还与公共点 (重合点) 数量和它们构成的几何形状结构息息 相关。

重合点选取原则是尽量选取足够的高等级、高精度且分布均匀的点作 为坐标转换的重合点 (重合点不少于 5 个)。为提高坐标转换精度, 必须使重 合点的数量足够多且分布均匀、合理, 同时还要经过大量的试算与分析剔 除一些变化大的重合点 (粗差点), 从而确定出最终用于求取转换参数的重 合点。

整理安庆市及其周边已有1954年北京坐标系、1980西安坐标系和2000 国家大地坐标系重合点, 对其进行分析, 选择精度高且分布均匀的控制点 作为坐标转换参数计算所用重合点。

3. 2 坐标转换模型。常用的坐标转换模型包括二维七参数模型、布尔 莎七参数模型、二维四参数模型等。根据《国土资源2000国家大地坐标系 转换技术要求》, 省级以下的坐标转换可选择平面四参数模型。

安庆市 2000 国家大地坐标系转换项目拟采用平面四参数坐标转换模 型进行坐标转换。

平面四参数坐标转换模型:

$$
\left[\begin{array}{l}
x_{2} \\
y_{2}
\end{array}\right]=\left[\begin{array}{l}
x_{0} \\
y_{0}
\end{array}\right]+(1+m)\left[\begin{array}{cc}
\cos \alpha & -\sin \alpha \\
\sin \alpha & \cos \alpha
\end{array}\right]\left[\begin{array}{l}
x_{1} \\
y_{1}
\end{array}\right]
$$

其中, 2 个平移参数 $x_{0} 、 y_{0}, 1$ 个旋转参数 $\alpha$ 和 1 个尺度因子 $m$ 。

3. 3 坐标转换的具体步骤: (1) 将重合点坐标换算为高斯平面坐标; (2) 选取部分均匀分布的重合点 (不少 6 个) 作为外部检核点, 不参与转换参数 计算; (3) 将其余的重合点代入平面四参数模型求取转换参数, 再利用该转 


\title{
遥感影像水体信息的提取方法研究
}

\author{
郭兴平 梁彦平 \\ 华北地质勘查局五一九大队
}

DOI:10.32629/gmsm.v2i6.415

[摘 要] 根据遥感影像中不同光谱波段对不同地物的反射率特征,本文采用各种方法,对水体信息提取方式进行探索。主要包括以下几步,第一, 把获取到的遥感影像进行分割, 进而得到图像分析最小的单位一影响对象; 第二步, 识别并提取最小影像对象的特征, 构建出分类识别所需要的特 征向量; 最后,运用机器学习法,在遥感影像中,提取到所需要的水体信息。

[关键词] 高分辨率遥感影像; 图像分析; 水体信息提取

\section{引言}

本文的研究基础和对象是高分辨率遥感影像, 提取水体图像信息、运 用面向对象的办法。所包含的步骤分别是预先处理遥感影像、对遥感影像 进行分割、获取遥感影像的特征、水体信息的分类与辨识。

遥感影像的分割, 是为了获取影响对象, 为后续的特征提取、识别分类 提供最小的处理单元。预先处理遥感影像的原理是对遥感影像进行几何校 正, 并且, 对图像融合处理, 全面提高对象的精准程度和信息的正确性, 为 后期精准识别遥感影像打下基础。对水体信息进行分类与识别主要是设置 识别参数、寻找最优并仿真的途径。遥感影像的特征提取作用是构建多特 征组合的特征向量。

\section{1 遥感影像的预处理}

对于遥感影像的预先处理, 首先, 采用几何校正的方法, 构建并选取合 理的数学模型, 然后, 确定下标准地图, 大地控制点、原始畸变影像之间的 坐标关联关系将在这个过程中确定, 之后, 在相应规则的基础上, 进行重采 样, 最后就可以获得校正后的精确影像 ${ }^{[1]}$ 。

\section{1几何校正}

几何校正在遥感影像预处理中的主要步骤是, 采集大地控制点、构建 数学模型、重采样成像。其中, 构建数学模型的目的, 主要是为了模拟几何 畸变的产生过程, 校正遥感影像处理中的畸变。大地控制点的选取原则上 要选纹路较为清楚、容易定位并且特征鲜明的点, 比如, 位于交通主干道上 的十字路口、运动场地、山峰和微型的岛屿等。此外, 根据畸变的程度和

换参数回代, 求得重合点的转换坐标, 进而计算各重合点的坐标残差; (4) 根 据残差值大小, 若残差大于 3 倍中误差则剔除, 重新计算坐标转换参数; (5) 再重复第 (2)、(3) 步, 直到回代残差达到项目要求的转换精度为止。（6) 用计算的转换参数计算外部检核点的转换坐标, 并和已知坐标进行比较, 进行转换精度的外部检核。

注: 在求转换参数时不要只追求回代精度, 同时也应考虑结构和图形 强度。

\section{4 质量控制措施}

4. 1 项目总体管理措施。项目按照 “两级检查、一级验收” 等相关的 测绘质量控制要求, 实行严格质量管理, 强化质量保障工作。加强组织和实 施质量监督管理, 制定质量监督管理办法, 加强项目承担单位的全面质量 管理, 加强对质量的跟踪监控。加强质量检查、质量评估、成果验收等工 作, 以保证项目的成果质量。

4. 2 坐标转换过程中的精度质量控制。坐标转换点位的平均精度应小 于图上的 $0.1 \mathrm{~mm}$ 。

坐标转换的精度是通过求取转换参数的重合点的残差中误差体现的。
校正精确度的要求, 可选取一阶多项式和二阶三阶多项式进行不同复杂程 度的校正。最近邻插值法是重采样成像的方式, 此方法用在邻域内最近的 像素值, 作为待采样点的像素值。公式如下图所示:

$$
\left\{\begin{array}{c}
k=\text { int eger }(x+0.5) \\
l=\text { int eger }(y+0.5) \\
f(x, y)=f(k, l)
\end{array}\right.
$$

其中, $(x, y)$ 为采样点的坐标, $(k, 1)$ 为最近的点的坐标。

1.2影像融合

多光谱影像与全色影像融合在一起, 可以同时具有空间分辨和光谱分 辨率的双重优势。图像融合技术能够很好的把不同的遥感器采集的多源 影像等信息重新融合在一起, 生成新的影像, 使影像具有更丰富更有效的 信息。

\section{2 遥感影像分割}

面向对象的办法, 能够克服噪声干扰造成光谱差异、影像纹理和几 何特征细节不明显的问题, 将图像分割获取来的影像对象分为最小的 处理单元。本次试验采用mean_shift方法可以提高后续继续分割的精 准度, 同时不会使图像看上去很模糊, 并可以充分的利用遥感影像的颜 色、形状、位置、纹理等细节。在分割前, 能够把获得的图像的随机噪 声去掉, 该方法操作十分简便、实用性很强, 能够显著提升遥感影像工 作的稳定性。

转换精度依据下式计算:

$\mathrm{v}($ 残差 $)=$ 重合点转换坐标 - 重合点已知坐

\section{5 结束语}

综上, 开展2000国家大地坐标系转换是我国地理测绘事业的发展所需, 为有效推进安庆市地理信息数据的应用与共享, 提高地理信息数据服务水 平, 需要将原有坐标系地理信息数据转换到2000国家大地坐标系。在转换 时, 应当结合转换任务, 科学设置观测点的数量及位置, 将所观测的数据进 行数据转换, 同时做好质量控制, 保障转换精度、减少误差, 从而确保大地 坐标系转换工作的高效开展。

\section{[参考文献]}

[1]宁左通.数字正射影像图向2000国家大地坐标系转换的原理和方 法研究[J].居舍,2019,(23):172+109.

[2]彭楚峡.2000国家大地坐标系转换方法研究 [J]. 住宅与房地 产,2019,(21):255.

[3]丁庆福,周晓,孙栋.市级2000国家大地坐标转换[J].科技创新导 报,2019,16(08):151-152. 